\title{
THE THEOREM OF IDENTITY FOR COHERENT ANALYTIC MODULES
}

\author{
HIROTAKA FUJIMOTO
}

To Professor Kiyoshi Noshiro on the occasion of his 60 th birthday

1. Introduction. The theorem of identity for analytic subsets of a reduced complex space is stated as follows;

Let $V$ and $V^{\prime}$ be two analytic subsets of a reduced complex space. If $V$ is irreducible and there exists a point $x \in V$ such that the germ $V_{x}$ of $V$ at $x$ is included in $V_{x}^{\prime}$, then $V$ is included in $V^{\prime}$.

To an analytic set $V$ there corresponds exactly one coherent Ideal $\mathscr{A}$ with $\operatorname{rad} \mathscr{A}=\mathscr{A}$. Replacing the above statement concerning analytic sets by coherent Ideals, we obtain the theorem of identity for coherent Ideals with suitable conditions. The first result of this paper is to generalize this to the case of coherent Modules over a complex space $X=(|X|, \mathcal{O})$.

Let $\mathscr{L}$ be an arbitrarily given coherent $\mathscr{O}$-Module over $X$. We shall call a coherent sub-O $\mathcal{O}$-Module $\mathscr{M}$ of $\mathscr{L}$ over $X$ to be primary if $\mathscr{H} x$ has no embedded primary component for any $x \in|\mathscr{L}| \mathscr{M} \mid:=\left\{x ;(\mathscr{L} / \mathscr{M})_{x} \neq 0\right\}$ and the analytic subset $|\mathscr{L}| \mathscr{K} \mid$ is irreducible.

We shall prove in $\S 3$

TheOREM (1st theorem of identity). If $\mathscr{M}$ is primary and $\mathscr{K}_{x} \supseteqq \mathscr{N}_{x}$ for another coherent sub-O-Module $\mathscr{N}$ of $\mathscr{L}$ and some $x \in|\mathscr{L}| \mathscr{M} \mid$, we have $\mathscr{M} \supseteqq \mathscr{N}$.

For an arbitrary $\mathscr{M}$, we shall show

THEOREM (2nd theorem of identity). For an arbitrarily given coherent subO-Module $\mathscr{M}$ of $\mathscr{L}$ there exists a locally finite family of irreducible analytic sets $\left\{V_{\iota}\right\}$ such that any coherent sub-0O-Module $\mathscr{N}$ of $\mathscr{L}$ with $\left\{x ; \mathscr{M}_{x} \supseteqq \mathscr{N}_{x}\right\} \cap V_{\iota} \neq \phi$ for any $\mathrm{s}$ is contained in $\mathscr{M}(\S 5)$.

To seek such $\left\{V_{\iota}\right\}$ we pay attention to a reduced primary decomposition of $\mathscr{M}_{x}$ in $\mathscr{L}_{x}$ for $x \in|\mathscr{L} / \mathscr{M}|$. The class of all prime ideals associated with

Received May 4, 1966. 
$\mathscr{M}_{x}$ is uniquely determined independently of a particular reduced primary decomposition considered. By piecing together these prime ideals we shall construct the coherent Ideals which define desired analytic sets $V$ 's $(\$ 4)$.

In a reduced primary decomposition an isolated primary component is also uniquely determined. The same argument as the above is applicable to costruct the isolated primary components of a coherent Module $\ddot{K}$. We shall obtain coherent primary Modules by piecing together isolated components of each $\mathscr{M}_{x}$. Using this, if each $\mathscr{M}_{x}$ has no embedded primary component in $\mathscr{L}_{x}$, we shall show $\mathscr{M}$ can be represented as the intersection of a locally finite family of primary Modules. In case that $X$ is Stein, such a coherent primary sub-OOC. Module $\mathscr{M}$ of $\mathscr{L}$ defines just a closed primary $H^{0}(X, \mathscr{O})$-submodule $H^{0}(X, \mathscr{M})$ of a Stein module $H^{0}(X, \mathcal{O})$ in [6]. Our primary decomposition theorem is a generalization of Forster's theorem ([6] p. 328) to the case of arbitrary complex spaces.

As an application of these results, we can prove Abhyankar's theorem ([1]) concerning the continuity of order on a complex space without the assumption of the simplicity of irreducible analytic set germs (Theorem 7.4). Moreover, we shall show that for a normal complex space $X$ there exists a canonical bijection between the set of all positive divisors on $X$ and the set of coherent Ideals with suitable conditions $(\S 8)$.

The auther would like to thank Mr. T. Okano for his many useful sug. gestions.

2. Algebraic preliminaries. Let $R$ be a commutative noetherian ring with identity and $L$ be an $R$-module of finite type.

An $R$-submodule $Q$ of $L(Q \varsubsetneqq L)$ is called to be primary if it satisfies the condition that for any $a \in R$ and $m \in L$ the conditions $a m \in Q$ and $m \notin Q$ imply that $a^{n} \cdot L \leqq Q$ for a suitable positive integer $n$. For a primary $R$-submodule $Q$ of $L$, the ideal $Q: L=\{a \in R ; a \cdot L \leqq Q\}$ is a primary ideal in $R$. We shall call $\operatorname{rad}(Q: L)$ the prime ideal associated with $Q$.

As is well known, every $R$-submodule $M$ of $L(M \nsubseteq L)$ has a primary decomposition

$$
M=Q_{1} \cap \cdots \cap Q_{s}
$$

where each $Q_{i}$ is a primary $R$-submodule of $L$. By $p_{i}$ we denote the prime 
ideal associated with $Q_{i}$ in this decomposition. Omitting some of $Q_{i}$ in the

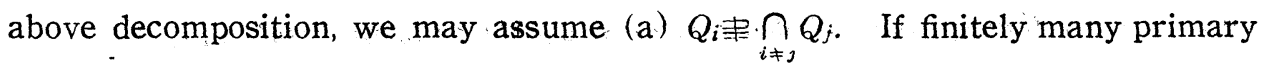
$R$-submodules $Q_{i}$ of $L$ define the same prime ideal $\mathfrak{p}:=\operatorname{rad}\left(Q_{i}: L\right)$, then $\cap_{i} Q_{i}$ is also an primary $R$-submodule of $L$. Using this, in (P) we may also assume (b) $\mathfrak{p}_{i} \neq \mathfrak{p}_{j}(i \neq j)$. We shall call a primary decomposition satisfying these conditions (a) and (b) a reduced primary decomposition of $M$ in $L$.

(2.1) Let $M=Q_{1} \cap \cdots \cap Q_{s}=Q_{1}^{\prime} \cap \cdots \cap Q_{t}^{\prime}$ be two reduced primary decompositions and $p_{i}(1 \leqq i \leqq s), p_{j}^{\prime}(1 \leqq j \leqq t)$ be the prime ideals associated with $Q_{i}, Q_{j}^{\prime}$ respectively. Then the sets $\left\{p_{i}\right\}$ and $\left\{p_{j}^{\prime}\right\}$ coincide with each other. We denote it by $\operatorname{Ass}_{L}(M)$. For convenience' sake we put $\operatorname{Ass}_{L}(M)=\phi$ if $L=M$.

Each $Q_{i}$ corresponding to a minimal $p_{i}$ in $\operatorname{Ass}_{L}(M)$ is called an isolated component of $M$ in $L$ and the other $Q_{j}$ is called an embedded component.

Let $S$ be a multiplicatively closed subset of $R$. For an $R$-submodule $M$ of $L$, we consider the set $M_{L}[S]:=\{m \in L ; s m \in M$ for some $s \in S\}$ and call it the $S$-component of $M$.

(2.2) If $M$ has a primary decomposition $(P)$ and $S \cap n_{i}=\phi(1 \leqq i \leqq t)$, $S \cap p_{j} \neq \phi(t+1 \leqq j \leqq s)$ for prime ideals $p_{i}$ associated with $Q_{i}$, then we have

$$
M_{L}[S]=Q_{1} \cap \cdots \cap Q_{t}
$$

Now, we take a subset $\mathbf{P}$ of $\operatorname{Ass}_{\llcorner}(M)$. If $\mathbf{P}$ satisfies the condition that any $\mathfrak{p} \in \operatorname{Ass}_{L}(M)$ with $\mathfrak{p} \cong p^{\prime}$ for some $p^{\prime} \in \mathbf{P}$ is always contained in $\mathbf{P}$, we shall call it an isolated subset.

(2.3) Let $M=Q_{1} \cap \cdots \cap Q_{s}$ be a reduced primary decompositon of $M$ in $L$ and $p_{i}$ be the prime ideals associated with each $Q_{i}$. Assume that a set $\mathbf{P}=\left\{p_{i_{1}}, \ldots, p_{i_{t}}\right\}$ is an isolated subset of $\left\{p_{i}\right\}$. Then $\cap \leqq Q_{1 \leqq l} Q_{i_{\nu}}$ depends only on $\mathbf{P}$ and not on a particular reduced primary decomposition, because we have $M_{L}[S]=\bigcap_{1 \leqq \nu \triangleq t} Q_{i_{\nu}}$ for the multiplicatively closed set $S=\bigcap_{1 \leqq \nu \leqq t}\left(R-\mathfrak{p}_{i_{\nu}}\right)$. We shall call it the P-component of $M$ and denote it by $M_{L}[\mathrm{P}]$ frequently.

Particularly, a minimal element $\mathfrak{p}$ in $\operatorname{Ass}_{L}(M)$ defines an isolated subset $\mathbf{P}=\{p\}$. The $\mathbf{P}$-component, or simply $p$-component of $M$ is nothing but an isolated component of $M$ defined by $\mathfrak{p}$, which depends only on $\mathfrak{p}$.

Let $M$ and $N$ be two $R$-submodules of $L . \quad$ It is a necessary and sufficient condition for $M \geqq N$ that the ideal $M: N$ in $R$ is equal to the total ring $R$.

For later uses, we give 
LEMMA 2.4. Under the above situation, the radical of the ideal $M: N$ is equal to the intersection of some of elements in $\operatorname{Ass}_{L}(M)$ or to the total ring $R$.

Proof. Take a reduced primary decomposition

$$
M=Q_{1} \cap \cdots \cap Q_{s}
$$

Easily, we have

$$
\operatorname{rad}(M: N)=\operatorname{rad}\left(Q_{1}: N\right) \cap \cdots \cap \operatorname{rad}\left(Q_{s}: N\right) .
$$

To complete the proof, it suffices to show (a) $\operatorname{rad}\left(Q_{i}: N\right)=R$ if $N \cong Q_{i}$ and (b) $\operatorname{rad}\left(Q_{j}: N\right)=\mathfrak{p}_{j}$ for the prime ideal $\mathfrak{p}_{j}$ associated with $Q_{j}$ if $N \$ Q_{j}$. The condition (a) is evident. To see (b), we assume $N \$ Q_{j}$, namely, there exists an element $n \in N$ with $n \notin Q_{j}$. Let $a$ be an arbitrary element in $Q_{j}: N$. Since $a n \in Q_{j}$ and $n \notin Q_{j}$, we see $a \in p_{j}=\operatorname{rad}\left(Q_{j}: L\right)$ by the definition of a primary module. This implies $Q_{j}: N \cong \mathfrak{p}_{j}$ and therefore $\operatorname{rad}\left(Q_{j}: N\right) \cong \mathfrak{p}_{j}$. On the other hand, evidently $\operatorname{rad}\left(Q_{j}: N\right) \supseteqq \operatorname{rad}\left(Q_{j}: L\right)=\mathfrak{p}_{j}$. Thus we conclude rad $\left(Q_{j}: N\right)=\mathfrak{p}_{j}$.

q.e.d.

3. The first theorem of identity. Let $X=\left(|X|, \mathscr{O}_{x}\right)$ be a complex space in the sense of Grauert [7] with the basic topological space $|X|$ and the structure sheaf $\mathscr{O}_{x}$, or simply $\mathcal{O}$. Take an arbitrary coherent analytic sheaf $\mathscr{L}$ over $X$. In the following, we restrict ourselves to the study of coherent analytic sheaves of $\mathscr{L}$ over $X$. Following Grothendieck [8], by a coherent

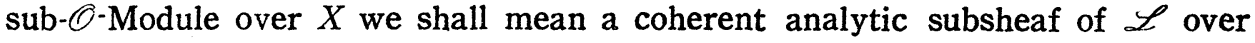
$X$. Particularly, if $\mathscr{L}=\mathscr{O}$, by a coherent Ideal we shall mean a coherent analytic subsheaf of $\mathscr{O}$ i.e. a coherent analytic sheaf of ideals.

A coherent Ideal $\mathscr{A}$ defines exactly one closed complex subspace $(|\mathscr{O} / \mathscr{A}|$, $\mathscr{C} / \mathscr{A})$ of $X$, where $|\mathcal{O} / \mathscr{A}|$ denotes the subset $\left\{x \in|X| ;(\mathscr{O} / \mathscr{A})_{x} \neq 0\right\}$ of $|X|$.

Now we recall the coherency of the Ideal $\operatorname{rad}(0)$ of $\mathcal{O}$. The complex subspace $(|X|, C / \operatorname{rad}(0))$ of $X$ is called the reduction of $X$ and denoted by red $X$. By defini.ion, a reduced complex space $X$ is a complex space with $X=\operatorname{red} X$. By an analytic subset of $X$ we shall mean the basic topological space for a reduced complex subspace of $X$ without consideration of the structure sheaf, and by a reduced irreducible complex space we shall mean a reduced complex space which cannot be decomposed into the union of two proper analytic subsets of $X$. 
Definition 3.1. A coherent sub-OOOModule $\mathscr{M}$ of $\mathscr{L}$ is called primary if it satisfies the followings ;

(i) for any $x \in|\mathscr{L} / \mathscr{M}|$ a reduced primary decomposition of $\mathscr{M}_{x}$ in $\mathscr{L}_{x}$ has no embedded component,

(ii) the set $|\mathscr{L}| \mathscr{M} \mid$ is an irreducible analytic subset of $X$.

In connection with coherent Modules, we give the following theorem which is a generalization of the theorem of identity for analytic subsets of a reduced complex space.

Theorem 3.2. Let $\mathscr{M}$ and $\mathscr{A}$ be two coherent sub-OO-Modules of $\mathscr{L}$ over $X$. If $\mathscr{M}$ is primary and there exists at least one point $x_{0} \in|\mathscr{L}| \mathscr{M} \mid$ such that $\mathscr{M}_{x_{0}} \supseteqq \mathscr{N}_{x_{0}}$, then it holds $\mathscr{M} \supseteqq \mathscr{N}$, or $\mathscr{M}_{x} \supseteqq \mathscr{N}_{x}$ for any $x \in|X|$.

Proof. Since $\mathscr{M}: \mathscr{L} \subseteq \mathscr{M}: \mathscr{N}$, the complex subspace $V:=(|\mathcal{O}| \mathscr{M}: \mathscr{N} \mid$, $\mathscr{O} / \mathscr{M}: \mathscr{N})$ of $X$ is also a complex subspace of $V^{\prime}=(|\mathcal{C}| \mathscr{M}: \mathscr{L}|, \mathscr{C}| \mathscr{M}: \mathscr{L})$. Therefore $|V|=|\mathscr{O}| \mathscr{M}: \mathscr{N} \mid$ is an analytic subset of $V^{\prime}$. We assume $|V| \neq \phi$ and take a point $y$ in $|V|$. By Lemma 2.4, $\operatorname{rad}\left(\mathscr{M}_{y}: \mathscr{N}_{y}\right)$ is equal to the intersection of a subset of $\operatorname{Ass}_{x_{y}}\left(\mathscr{M}_{y}\right)$. This shows the germ $|V|_{y}$ of $|V|$ at $y$ is the union of some of irreducible components of $\left|V^{\prime}\right|_{y}$ by virtue of the condition (i) of Definition 3.1. Now, we can apply the theorem of identity for an irreducible analytic subset $\left|V^{\prime}\right|$ of red $X$. Thus we have $|V| \subseteq\left|V^{\prime}\right| \subseteq|V|$ and so $|V|=\left|V^{\prime}\right|$. On the other hand, the point $x_{0}$ is contained in $\left|V^{\prime}\right|$ but not in $|V|$ by the assumption. This is a contradiction. We conclude $|V|=\phi$, which shows $\mathscr{M}: \mathscr{N}=\mathscr{C}$, namely, $\mathscr{M} \supseteqq \mathscr{N}$.

q.e.d.

We can weaken slightly the assumption of Theorem 3.2 as follows.

Corollary 3.3. Under the same situation in Theorem 3.2, if $\mathscr{M}$ is primary and there exists a point $x_{0} \in|\mathscr{L}| \mathcal{M} \mid$ such that for a primary component $Q$ of $\mathscr{M}_{x_{0}}$ in $\mathscr{L}_{x_{0}} Q \supseteqq \mathscr{T}_{x_{0}}$, then it holds $\mathscr{M} \supseteqq \mathscr{N}$.

Proof. Let $\mathscr{M}_{x_{0}}=Q_{1} \cap \cdots \cap Q_{s}$ be a reduced primary decomposition of $\mathscr{M}_{x_{0}}$ in $\mathscr{L}_{x_{0}}$, say $Q_{1}=Q$. Since $\mathscr{L}_{x_{0}}$ is an $\mathscr{C}_{x_{0}}$-module of finite type, there exists a finite system of generators of an $C_{x_{0}}$-module $Q_{i}$ for each $Q_{i}$. Using these generators, we can define coherent sub-Ci-Module $\mathscr{Q}_{i}$ of $\mathscr{L}$ over a suitable neighborhood $U$ of $x_{0}$ such that

$$
\mathscr{M}=\mathscr{Q}_{1} \cap \cdots \cap \mathscr{Q}_{s}
$$


on $U$. Then, making $U$ sufficiently small, we can assume $\mathscr{Q}_{1} \supseteq \mathscr{N}$ on $U$ because $\mathscr{Q}_{1 x_{0}}=Q \supseteqq \mathscr{N}_{x_{0}}$. On the other hand, by the condition (i) of Definition 3.1 the prime ideal $\mathfrak{p}_{i}$ associated with $Q_{i}$ satisfies $p_{1} \bigcap_{j \neq 1} p_{j}$. It follows from Nullstellensatz (c.f. [4] Exposé 19, p. 17) there exists a point $y \in\left|\mathscr{L} / \mathscr{Q}_{1}\right|$ such that $y \notin|\mathscr{L}| \mathscr{Q}_{i} \mid$ for any $i \neq 1$. Then we see easily $y \in|\mathscr{L}| \mathscr{M} \mid$ and $\mathscr{M}_{y}=\mathscr{Q}_{1_{y}} \supseteq \mathscr{N}_{y}$. This shows $\mathscr{M}$ and $\mathscr{N}$ satisfy the conditions in Theorem 3.2. The proof is accomplished.

q.e.d.

Corollary 3.4. Let $\mathscr{M}$ be a coherent primary sub-OO-Module of $\mathscr{L}$. If a section $f \in H^{0}(X, \mathscr{L})$ satisfies $f_{x_{0}} \in Q$ for a point $x_{0} \in|\mathscr{L}| \mathscr{M} \mid$ and a primary component $Q$ of $\mathscr{M}_{x_{0}}$, then $f$ is contained in $H^{0}(X, \mathscr{M})$.

Proof. Putting $\mathscr{N}_{x}:=\mathscr{O}_{x} \cdot\left\{f_{x}\right\}$ in $\mathscr{L}_{x}$ for each $x \in|X|$, we get a coherent sub-OO-Module $\mathscr{N}=\bigcup_{x \in|X|} \mathscr{N}_{x}$ of $\mathscr{L}$ over $X$. Apply Corollary 3.3 to the coherent Modules $\mathscr{M}$ and $\mathscr{N}$. We have $f \in H^{0}(X, \mathscr{N}) \subseteq H^{\circ}(X, \mathscr{M})$.

A coherent sub- $\mathscr{O}$-Modules $\mathscr{M}$ of $\mathscr{L}$ defines an $H^{0}(X, \mathscr{O})$-submodule of $H^{0}(X, \mathscr{L})$.

Corollary 3.5. If $\mathscr{M}$ is primary, then $H^{0}(X, \mathscr{M})$ is a primary submodule of $H^{0}(X, \mathscr{L})$, that is, for any $f \in H^{0}(X, \mathcal{O})$ and $g \in H^{0}(X, \mathscr{L})$ the condition $f g \in H^{0}(X, \mathscr{M})$ and $g \notin H^{0}(X, \mathscr{M})$ implies $f^{n} H^{0}(X, \mathscr{L}) \leqq H^{0}(X, \mathscr{M})$ for a suitable positive integer $n$.

Proof. We take a point $x \in V:=|\mathscr{L}| \mathscr{M}|=| \mathcal{O}|\mathscr{M}: \mathscr{L}|$ such that the analytic subset $V$ of $X$ is irreducible at $x$. For a reduced primary decomposition $\mathscr{M}_{x}=Q_{1} \cap \cdots \cap Q_{s}$, we have

$$
\mathscr{M}_{x}: \mathscr{L}_{x}=\left(Q_{1}: \mathscr{L}_{x}\right) \cap \cdots \cap\left(Q_{s}: \mathscr{L}_{x}\right),
$$

which is a primary decomposition of the ideal $\mathscr{M}_{x}: \mathscr{L}_{x}$. This shows Ass ${ }_{0_{x}}$ $\left(\mathscr{M}_{x}: \mathscr{L}_{x}\right) \subseteq \operatorname{Ass}_{\mathscr{Z}_{x}}\left(\mathcal{L} \mathscr{M}_{x}\right)$ and any minimal element in $\operatorname{Ass}_{Z_{x}}\left(\mathscr{M}_{x}\right)$ is contained in $\operatorname{Ass}_{\jmath_{x}}\left(\mathcal{M}_{x}: \mathscr{L}_{x}\right)$. By the irreducibility of $V_{x} \operatorname{Ass}_{\vartheta_{x}}\left(\mathscr{M}_{x}: \mathscr{L}_{x}\right)$ contains only one minimal element and by the assumption of $\mathscr{M}_{x}$ all elements of Ass $_{\mathcal{L}_{x}}\left(\mathscr{M}_{x}\right)$ are minimal. Therefore, $\operatorname{Ass}_{\mathcal{L}}\left(\mathscr{M}_{x}\right)$ consists of only one prime ideal and so $\mathscr{M}_{x}$ is primary in $\mathscr{L}_{x}$.

On the other hand, by the assumption of $g \notin H^{0}(X, \mathscr{M})$, there exists a point $y$ such that $g_{y} \notin \mathscr{M}_{y}$. Moreover, according to Corollary 3.4 , we can assert $g_{z} \notin \mathscr{M}_{z}$ for any $z$ in $|\mathscr{L}| \mathscr{M} \mid$, especially the above $x$. Thus we see 
$f_{x} g_{x} \in M_{x}$ and $g_{x} \notin \mathscr{M}_{x}$ for a primary module $\mathscr{M}_{x}$ in $\mathscr{L}_{x}$ and so $f_{x}^{n} \cdot \mathscr{L}_{x} \cong \mathscr{M}_{x}$ for a suitable positive integer $n$ by definition. Applying Corollary 3.4 again we have $f^{n} h \in H^{0}(X, \mathscr{M})$ for any $h \in H^{0}(X, \mathscr{L})$ because $\left(f^{n} h\right)_{x} \in \mathscr{M}_{x}$. Eventually, $f^{n} \cdot H^{0}(X, \mathscr{L}) \subseteq H^{0}(X, \mathscr{M})$, which completes the proof.

Remark. In $\S 6$, we shall consider the converse statement of Corollary 3.5 (see Theorem 6.5).

4. Coherent prime Ideals associated with a coherent Module. A coherent sub-C-Module $\mathscr{M}$ of $\mathscr{L}$ defines the set $\operatorname{Ass}_{\mathscr{L} x}\left(\mathscr{M}_{x}\right)$ of all prime ideals associated with $\mathscr{M}_{x}$ in $\mathscr{L}_{x}$ for each $x \in|X|((2.1))$. The purpose of this section is to introduce an analogous notion of coherent Modules from a global point of view.

Firstly, we give the following Lemma, which plays an essential role in this section.

Lemma 4.1. For a coherent sub-O-Module $\mathscr{M}$ of $\mathscr{L}$ over $X$ the set of all $x \in|X|$ such that $\mathscr{K}_{x}$ has no embedded component is an open subset of $|X|$.

This is a consequence of G. Scheja [13], especially, the assertion of the equivalence of the conditions (1) and (2) in Satz 9, p. 85 . Easily we can conclude the openness of the considered set in view of the condition (2) in it.

Remark. In case of $\mathscr{L}=\mathcal{O}$, we can give another proof of Lemma 4.1. For a coherent Ideal $\mathscr{A}$ and a point $x \in|X|, \mathscr{A}_{x}$ has no embedded component if and only if a ring $\mathscr{O}_{x} / \mathscr{A}_{x}$ satisfies Serre's condition $\left(S_{1}\right)$. On the other hand, the set of all $x \in|X|$ such that $\mathscr{O}_{x} / \mathscr{A}_{x}$ does not satisfy the condition $\left(S_{q}\right)$ is a closed analytic subset of red $X$ for any $q$ (c.f. Houzel [4] Exposé 21, p. 7, Proposition 4).

The following Lemma is a generalization of the assertions concerning primary decompositions stated in $\S 2$ to the case of coherent Modules under semi-local consideration.

Lemma 4.2. Let $\mathscr{M}$ be a coherent sub-O-Module of $\mathscr{L}$ over $X$. For any $x \in|\mathscr{L}| \mathscr{M} \mid$ we can find a sufficiently small neighborhood $U$ of $x$ and coherent primary sub-Ö-Modules $\mathscr{Q}_{i}(1 \leqq i \leqq s)$ of $\mathscr{L}$ satisfying the following conditions;

(i) $\mathscr{M}=\mathscr{Q}_{1} \cap \cdots \cap \mathscr{Q}_{s}$ on $U$.

(ii) each $\mathscr{Q}_{i x}$ is a primary $\mathscr{O}_{x}$-submodule of $\mathscr{L}_{x}$,

(iii) for any $y \in U \cap|\mathscr{L}| \mathscr{M} \mid$, if we take an arbitrary reduced primary 
decomposition of each $\mathscr{Q}_{i y}$ in $\mathscr{L}_{y}$

$$
\mathscr{Q}_{i y}=Q_{i, 1} \cap \cdots \cap Q_{i, k_{i}},
$$

then the primary decomposition of $\mathscr{M}_{y}$

$$
\mathscr{M}_{y}=\cap_{i}\left(Q_{i, 1} \cap \cdots \cap Q_{i, k_{z}}\right)
$$

is also a reduced primary decomposition, where we consider the indices $i$ with $\mathscr{Q}_{i y} \neq \mathscr{L}_{y}$ only in the above.

Proof. We take a reduced primary decomposition

$$
\mathscr{M}_{x}=Q_{1} \cap \cdots \cap Q_{s},
$$

where each $Q_{i}$ is considered as the stalk of a coherent Module $\mathscr{Q}_{i}$ over a sufficiently small neighborhood $U$ of $x$ by the same argument in the proof of Corollary 3.3. Then, it may be assumed

$$
\mathscr{M}=\mathscr{Q}_{1} \cap \cdots \cap \mathscr{Q}_{s}
$$

on $U$ and, moreover, according to Lemma 4.2 each $\mathscr{Q}_{i y}$ may be assumed to have no embedded component for any $y \in U \cap|\mathscr{L}| \mathscr{Q}_{i} \mid$. For our purpose, it suffices to take these $U$ and $\mathscr{Q}_{i}(1 \leqq i \leqq s)$. The conditions (i) and (ii) are satisfied automatically. To examine the condition (iii), it is necessary to show (a) $Q_{i, k}$ 声 $\bigcap_{(i, k) \neq 1, j, l} Q_{j, l}$ and (b) $\mathfrak{p}_{i, k}:=\operatorname{rad}\left(Q_{i, k}: \mathscr{L}_{y}\right) \neq p_{j, l}:=\operatorname{rad}\left(Q_{j, l}: \mathscr{L}_{y}\right)$ for any $(i, k) \neq(j, l)$, where $Q_{i y}=\bigcap_{1 \leqq l \leqq k_{i}} Q_{i, l}$ as in the condition (iii). Firstly, we apply Corollary 3.3 to the coherent sub-CO-Modules $\mathscr{Q}_{i}$ and $\bigcap_{i \neq j} \mathscr{Q}_{j}$ of $\mathscr{L}$ over $U$. Since $\mathscr{Q}_{i}$ is primary over $U$ and $\mathscr{Q}_{i x} \underset{i \neq j}{\bigcap} \mathscr{Q}_{j x}$, any primary component $Q_{i, l}$ of $\mathscr{Q}_{i y}$ does not include $\bigcap_{i \neq j} \mathscr{Q}_{j y}=\bigcap_{i \neq j} \bigcap_{1 \leqq l \leqq k_{j}} Q_{j, l}$. Therefore, we can take an element $f \in \bigcap_{i \neq j} \bigcap_{1 \leqq l \leqq k_{j}} Q_{j_{1}}$, with $f \notin Q_{i, k}$. Since $\mathfrak{p}_{i, k}$ is a minimal element in $\operatorname{Ass}_{\not y}\left(\mathscr{Q}_{i y}\right)$, we can take also an element $g \in \bigcap_{k \neq l} \mathfrak{p}_{i, l}$ with $g \notin \mathfrak{p}_{i, k}$. Replacing $g$ by $g^{n}$ for a suitable $n$ if necessary, we can assume $g \in \bigcap_{k \neq l}\left(Q_{i, l}: \mathscr{L}_{y}\right)$ and $g \notin p_{i, k}$. Then the element $h=g \cdot f$ is contained in $\bigcap_{(i, k) \neq(j, l)} Q_{j, l}$ but not in $Q_{i, k}$. This asserts (a). On the other hand, by Definition 3.1, each $V_{i}=\left|\mathscr{L}_{y}\right| \mathscr{Q}_{i} \mid$ is an irreducible analytic subset of $U$ and defines exactly one irreducible component of $V_{i x}$. Now the assertion (b) is an immediate consequence of the theorem of identity for analytic subsets of a reduced complex space. $\quad$ q.e.d.

We shall call a coherent Ideal $\mathscr{P}$ over $X$ to be prime if it defines a reduced irreducible subspace $(|\mathscr{C} / \mathscr{P}|, \mathscr{C} / \mathscr{P})$ of $X$. In view of the proof of Lemma 
4.2, for each $x \in|X|$ there exists a neighborhood $U$ of $x$ and coherent prime Ideals $\mathscr{M}_{i}(1 \leqq i \leqq s)$ such that $\cup_{i} \operatorname{Ass}_{\mathcal{O}_{y}}\left(\mathscr{P}_{i y}\right)=\operatorname{Ass}_{\mathcal{L}_{y}}\left(\mathscr{M}_{y}\right)$ for any $y \in U$. The following theorem asserts this is valid globally.

Theorem 4.3. For an arbitrary coherent sub-CO-Module $\mathscr{M}$ of $\mathscr{L}$ over $X$, there exists one and only one family $\left\{\mathscr{P}_{c}:, \in I\right\}$ of coherent prime Ideals over $X$ such that $\bigcup_{\iota \in I} \operatorname{Ass}_{\mathscr{O}_{y \prime}}\left(\mathscr{P}_{\iota_{y}}\right)=\operatorname{Ass}_{\mathcal{L}_{y}}\left(\mathscr{M}_{y}\right)$ for any $y \in|X|$. We shall call it the set of all coherent prime Ideals associated with $\mathscr{M}$ and denote it by $\mathbf{A s s _ { Z }}(\mathscr{M})$.

Proof. To see the uniqueness, we have only to recall the theorem of identity, which asserts any two distinct coherent prime Ideals $\mathscr{P}$ and $\mathscr{P}$, satisfy $\operatorname{Ass}_{\mathcal{O}_{x}}\left(\mathscr{P}_{x}\right) \cap \operatorname{Ass}_{\mathscr{D}_{x}}\left(\mathscr{P}_{x}^{\prime}\right)=\phi$ for any $x \in|X|$.

For each non-negative integer $k$, we define the set $V_{k}$ of all points $x$ such that there exists at least one prime ideal of depth $k$ in $\operatorname{Ass}_{\mathscr{L} x}\left(\mathcal{M}_{x}\right)$. We want to show each $V_{k}$ is an analytic subset of $X$. Obviously, $V_{k}$ is analytic at a point $x \notin|\mathscr{L}| \mathscr{M} \mid$. We take a point $x \in|\mathscr{L} / \mathscr{M}|$. By the above note, there exists a neighborhood $U$ of $x$ and coherent prime Ideals $\mathscr{P}_{i}(1 \leqq i \leqq s)$ satisfying the conditions in Theorem 4.3 for the coherent Module $\mathscr{M} \mid U$ over $U$. By the uniqueness of the prime ideals associated with $\mathscr{M}_{x}$, we can easily prove $V_{k} \cap U$ is the union of all $|\mathscr{O}| \mathscr{P}_{i} \mid$ with $\mathscr{P}_{i} \in \operatorname{Ass}_{\mathscr{L}}(\mathscr{M})$ of depth $k$. Consequently, $V_{k}$ is analytic at $x$ in this case too. By definition, for any $y \in|\mathscr{L} / \mathscr{M}|$ any irreducible component of $V_{k}$ is an analytic set defined by some $\mathfrak{p} \in \operatorname{Ass}_{Z_{y}}\left(\mathscr{M}_{y}\right)$ of depth $k$ and conversely any $p \in \operatorname{Ass}_{z_{y}}\left(\mathscr{M}_{y}\right)$ defines an irreducible component of $V_{k y}$ if $k=$ depth $p$. If we take the prime Ideal $\mathscr{P}_{k c}$ corresponding to each irreducible component $V_{k \text { s }}$ of each $V_{k}$, the family $\left\{\mathscr{g}_{k \ell}\right\}$ satisfies the desired condition.

q.e.d.

Remark. The above set $\operatorname{Ass}_{\mathcal{L}}(\mathscr{M})$ is always a locally finite family of prime Ideals, that is, the family $\left\{|\mathscr{O}| \mathscr{P} \mid ; \mathscr{P} \in \operatorname{Ass}_{Z}(\mathscr{M})\right\}$ is a locally finite family of closed subsets of $|X|$. For, each point $x \in|X|$ has a neighborhood $U$ such that $\operatorname{Ass}_{\mathcal{L} \mid U}(\mathscr{M} \mid U)$ is finite and therefore the number of elements $\mathscr{P}$ 's in $\operatorname{Ass}_{\mathcal{L}}(\mathscr{M})$ with $|\mathscr{O} / \mathscr{P}| \cap U \neq \phi$ is finite.

5. The second theorem of identity: Using the results in $\S 4$, we can generalize Theorem 3.2 to arbitrary coherent Modules.

TheOREm 5.1. Let $\mathscr{M}$ and $\mathscr{A}$ be coherent sub-CO-Modules of $\mathscr{L}$ over $X$. If $\left\{x ; \mathscr{M}_{x} \supseteqq \mathscr{T}_{x}\right\}$ intersects the analytic subset $V_{l}:=|\mathscr{L}| \mathscr{P}_{b} \mid$ of $X$ for any 
$\mathscr{P}_{\iota} \in \operatorname{Ass}_{\mathcal{L}}(\mathscr{M})$, then it holds $\mathscr{M} \supseteqq \mathscr{N}$.

Proof. The proof is similar to Theorem 3.2. Take a coherent sub-C-Module of $\mathscr{L}$. We consider the analytic subset $V:=|\mathscr{O} / \mathscr{M}: \mathscr{A}|$ of $X$. As in the proof of Theorem 3.2, Lemma 2.4 shows any irreducible component of $V_{y}$ is equal to an irreducible component of one of $\left\{V_{\iota y}\right\}$ for each $y \in V$. Therefore, $V$ must be equal to the union of some of $\left\{V_{c}\right\}$ owing to the theorem of identity. On the other hand, by the assumption there exists a point $x_{\iota} \in V_{c}$ with $x_{\iota} \notin V$ for each c. Consequently, we conclude $V=\phi$ and so $\mathscr{M} \supseteqq \mathscr{Y}$.

q e d.

In the previous paper [5], we studied the continuability of analytic sets in a reduced complex space. To an analytic subset $V$ of a reduced complex space $X$ there corresponds one and only one coherent Ideal $\mathscr{A}:=\mathscr{F}(V)$ with $\mathrm{rad}$ $\mathscr{A}=\mathscr{A}$ consisting of all germs of holomorphic functions which vanish on $V$. We are interested in the study of the continuability of coherent Ideals without the assumption $\operatorname{rad} \mathscr{A}=\mathscr{A}$ or more generally coherent Modules. Theorem 5.1 gives us a condition for the uniqueness of continuability of coherent Modules as follows (c.f. Corollary 8.2).

For convenience' sake, we give

Definition 5.2. For a coherent sub-OOC.Module $\mathscr{M}$ over $X$ we shall say $\mathscr{M}$ to be of lower dimension $k$ in $\mathscr{L}$ at $x$ if $1 . \operatorname{dim}_{\mathcal{L}_{x}} \mathscr{M}_{x}:=\min \left\{\operatorname{dim} \mathscr{O}_{x} / \mathfrak{p}\right.$; $\left.\mathfrak{p} \in \operatorname{Ass}_{\mathcal{L}_{x}}\left(\mathscr{M}_{x}\right)\right\}=k$ and to be of lower dimension $k$ on $X$ if $1 \operatorname{dim}_{\mathcal{L}} \mathscr{K}:=\min _{x \in|X|}$ $1 \cdot \operatorname{dim}_{\mathcal{Z} x} \mathscr{M}_{x}=k$, where we put $1 \operatorname{dim}_{\mathcal{Z}_{3}}\left(c \mathscr{M}_{x}\right)=\infty$ if $\operatorname{Ass}_{\mathcal{L} x}\left(\mathscr{M}_{x}\right)=\phi$.

Corollary 5.3. Let $X$ be a complex space and $X^{\prime}$ be an open subset of $|X|$ with the property that any analytic set of dimension $\geqq k$ in $X$ intersects $X^{\prime}$. If two coherent sub-O-Modules $\mathscr{M}$ and $\mathscr{N}$ satisfy the conditions $1 . \operatorname{dim}_{\mathcal{Z}} \mathscr{M} \geqq k$ and $\mathscr{M} \equiv \mathscr{N}$ on $X^{\prime}$, then it holds $\mathscr{M} \geqq \mathscr{N}$.

Furthermore, if $1 . \operatorname{dim}_{\mathcal{L}} \mathscr{N} \geqq k, \mathscr{M}=\mathscr{N}$ on $X^{\prime}$ implies $\mathscr{M}=\mathscr{N}$ on $X$.

Proof. By the assumption, the irreducible analytic subset of $X$ defined by each element of $\operatorname{Ass}_{\mathcal{L}}(\mathscr{M})$ is of dimension $\geqq k$ and therefore intersects $X^{\prime}$ by the assumption. We can easily examine the assumption of Theorem 5.1. q.e.d.

There are many well-known examples of complex spaces and their open sets satisfying the condition in Corollary 5.3. Here we give two typical examples.

Corollary 5.4. Let $X$ be a complex space, $V$ be an analytic subset of $X$ and $\mathscr{M}, \mathscr{N}$ be coherent sub-O-Modules of $\mathscr{L}$ over $X$. If $1 . \operatorname{dim}_{\mathcal{L x}} \mathscr{M}_{x}$ and $1 . \operatorname{dim}_{\mathcal{L}_{x}} \mathscr{N}_{x}$ 
are both larger than $\operatorname{dim}_{x} V$ for any $x \in V$, then $\mathscr{M}=\mathscr{N}$ on $|X|-V$ implies $\mathscr{M}=\mathscr{N}$ on $|X|$.

Indeed, any analytic set defined by $\mathscr{P} \in \operatorname{Ass}_{\mathcal{L}}(\mathcal{K})$ cannot be contained in $V$.

Corollary 5.5. Let $D$ be a bounded domain in $C^{n}$ and $M, \mathscr{N}$ be two coherent sub-Ci-Modules of a coherent $\mathscr{C}$-Module. L over a neighborhood of $\bar{D}$. If 1. $\operatorname{dim}_{\mathcal{L}} \mathbb{K}$ and $1 . \operatorname{dim}_{\mathscr{L}} \mathscr{N}$ are both positive and $\mathscr{M}=\mathscr{N}$ in a suitable neighborhood of the boundary of $D$, then we have $\mathscr{M}=\mathscr{N}$ on $D$.

Proof. We know that there is no compact analytic subset of positive dimension in $C^{n}$. Any analytic set of positive dimension in a neighborhood of $\bar{D}$ has to intersect $C^{n}-D$. Eventually, $\mathscr{M}$ and $\mathscr{N}$ satisfy all conditions in Corollary 5.3.

q.e.d.

The arguments used in the proof of Corollary 5.5 is applicable to a complex space which admits a function satisfying maximum principle on each $k$ dimensional analytic set e.g. a $*$-strongly $k$-convex function (see [5] $\S 2$ ).

6. Isolated components of coherent Modules. Let $\mathscr{M}$ be a coherent sub$\widehat{C}$-Module of $\mathscr{L}$ and $S$ be a multiplicatively closed set of holomorphic functions. We define a presheaf $\widetilde{\mathscr{M}[S]}$ by the assignment of the set

$$
\widetilde{\mathscr{M}}[\mathrm{S}](U):=\left\{f \in H^{0}(U, \mathscr{L}): s f \in H^{0}(U, \mathscr{M}) \text { for a suitable } s \in S\right\}
$$

to each open subset $U$ of $|X|$. By this presheaf $\widetilde{\mathscr{M}[S]}$, we obtain a subsheaf $\mathscr{M}[S]$ of $\mathscr{L}$ over $X$, whose stalk is $\mathscr{M}_{x}\left[S_{x}\right]$ at each $x \in|X|$.

Theorem 6.1. $\mathscr{M}[S]$ is a coherent Module over $X$.

Proof. The problem is local. It suffices to show each $x \in|\mathscr{L} / \mathscr{M}|$ has a neighborhood $U$ such that $\mathscr{M}[S]$ is a coherent Module over $U$. To see this, we take a neighborhood $U$ of $x$ and coherent primary Modules $\mathscr{Q}_{i}(1 \leqq i \leqq s)$ with the properties (i) $\sim$ (iii) in Lemma 4.2 over $U$. For any $y$ and any $i$, let $Q_{i, l}(y) \quad\left(1 \leqq l \leqq k_{i}(y)\right)$ be the primary component of $\mathscr{Q}_{i y}$. Now, we apply Corollary 3.5 to each $\mathscr{Q}_{i}$ and an arbitrarily fixed element of $S$. Easily, we see a necessary and sufficient condition for $S \cap H^{0}\left(U, \mathscr{P}_{i}\right) \neq \phi$ is that $S_{y} \cap \mathfrak{p}_{i, l}(y) \neq \phi$ for any $l$ and any $y \in U$, or that $S_{y} \cap p_{i, l}(y) \neq \phi$ for a particular $l$ and $y \in U$, where $\mathscr{P}_{i}=\operatorname{rad}\left(\mathscr{Q}_{i}: \mathscr{L}\right)$ and $\mathfrak{p}_{i, l}=\operatorname{rad}\left(Q_{i, l}: \mathscr{L}_{y}\right)$. Replacing indices suitably, 
we may assume $S \cap H^{0}\left(U, \mathscr{P}_{i}\right)=\phi$ for $1 \leqq i \leqq t$ and $S \cap H^{0}\left(U, \mathscr{P}_{j}\right) \neq \phi$ for $t+1 \leqq j \leqq s$. By (2.2), we conclude $\mathscr{M}[S]=\mathscr{Q}_{1} \cap \cdots \cap \mathscr{Q}_{t}$ on $U$, which is coherent over $U$. This completes the proof.

q.e.d.

Next, we generalize the notion of an isolated subset of $\operatorname{Ass}_{\mathcal{Z}}(\mathscr{M})$ to the case of coherent Modules.

Definition 6.2. For a coherent sub-CO-Module $\mathscr{M}$ of $\mathscr{L}$, we shall call a subset $I$ of $\operatorname{Ass}_{\mathscr{L}}(\mathscr{M})$ an isolated set if any $\mathscr{P} \in \operatorname{Ass}_{Z}\left(\mathscr{M}^{\prime}\right)$ with $\mathscr{P} \subseteq \mathscr{P}$ i for some $\mathscr{P}^{\prime} \in \Pi$ is always contained in $\Pi$.

Let $\Pi$ be an isolated subset of $\operatorname{Ass}_{\mathcal{L}}(\mathscr{M})$. For any $x \in|X|$, we consider the set $\Pi_{x}$ of all prime ideals associated with the stalk $\mathscr{P}_{x}$ of each $\mathscr{S}$ in $\Pi$. Each $\Pi_{x}$ is also an isolated subset of $\operatorname{Ass}_{\mathcal{L} x}\left(\mathscr{M}_{x}\right)$. Indeed, each prime ideal $\mathfrak{p} \in \operatorname{Ass}_{Z_{x}}\left(\mathscr{M}_{x}\right)$ is associated with only one $\mathscr{P} \in \operatorname{Ass}_{\mathcal{X}}(\mathscr{M})$ and this correspondence is order-preserving. Taking an element $\mathfrak{p} \in \operatorname{Ass}_{\mathcal{L} x}\left(\mathscr{M}_{x}\right)$ with $\mathfrak{p} \cong \mathfrak{p}^{\prime}$ for some $\mathfrak{p}^{\prime} \in \Pi_{x}$, the coherent prime Ideal $\mathscr{P}^{\prime}$ determined by $\mathfrak{p}^{\prime}$ includes the coherent prime Ideal $\mathscr{P}$ determined by $\mathfrak{p}$. By definition, $\mathfrak{p}^{\prime} \in \Pi_{x}$ implies $\mathscr{P} \mathfrak{P}^{\prime} \in \Pi, \mathscr{P} \in \Pi$ and hence $\mathfrak{p} \in \Pi_{x}$.

THEOREM 6.3. For an isolated subset II of Âss $\mathcal{L}_{\mathcal{L}}(\mathscr{M})$ there exists a coherent sub-O-O-Module $\mathscr{M}[\Pi]$ of $\mathscr{L}$ with the stalk $\mathscr{M}[\Pi]_{x}=\mathscr{M}_{x}\left[\Pi_{x}\right]$ for each $x \in|X|$.

We shall call it the II-component of $\mathscr{M}$.

Proof. As in the proof of Theorem 6.1, for an arbitrarily given point $x \in|\mathscr{L} / \mathscr{M}|$. we take coherent primary Modules over a suitable neighborhood $U$ of $x$ with the properties in Lemma 4.2. We see easily $\operatorname{Ass}_{\mathscr{L}_{y}}\left(\mathscr{Q}_{i y}\right) \cap \Pi_{y}=\phi$ for any $y \in U$ if it holds for at least one $y \in|\mathscr{L}| \mathscr{Q}_{i} \mid$. After a suitable change of indices we may assume $\operatorname{Ass}_{\mathscr{Z}_{y}}\left(\mathscr{Q}_{i y}\right) \cap \Pi_{y} \neq \phi$ for $1 \leqq i \leqq t$ and $\operatorname{Ass}_{\mathcal{Z}_{y}}\left(\mathscr{Q}_{j y}\right) \cap \Pi_{y}=\phi$ for $t+1 \leqq j \leqq s$. Since the former implies $\operatorname{Ass}_{\mathcal{L} y}\left(\mathscr{Q}_{i y}\right) \leqq \Pi$ for any $y \in|\mathscr{L}| \mathscr{Q}_{i} \mid$ and $\Pi_{y}$ is an isolated set, we have

$$
\mathcal{M}_{y}\left[\Pi_{y}\right]=\mathscr{Q}_{1 y} \cap \cdots \cap \mathscr{Q}_{t y} .
$$

Thus $\mathscr{M}[\Pi]:=\mathscr{Q}_{1} \cap \cdots \cap \mathscr{Q}_{t}$ is coherent over $U$. q.e.d.

Corollary 6.4. Suppose that for a coherent sub-O-Module $\mathscr{M}$ of $\mathscr{L}$ and each $x \in|\mathscr{L}| \mathscr{M} \mid \mathscr{M}_{x}$ has no embedded component. Then $\mathscr{M}$ is uniquely rep. resented as the intersection of a locally finite family $\left\{\mathscr{Q}_{\imath}\right\}$ of primary sub-OC-Modules 
of $\mathscr{L}$ such that $\mathscr{Q}_{\imath} \equiv \bigcap_{\iota \neq \iota^{\prime}} \mathscr{Q}_{\iota^{\prime}}$ and $\operatorname{rad}\left(\mathscr{Q}_{\imath}: \mathscr{L}\right) \neq \operatorname{rad}\left(\mathscr{Q}_{\imath^{\prime}}: \mathscr{L}\right)$ for $\imath \neq \imath^{\prime}$.

Proof. Any element $\mathscr{P}$ in $\operatorname{Ass}_{\mathcal{X}}(\mathscr{M})$ defines an isolated subset $\{\mathscr{P}\}$ of $\operatorname{Ass}_{\mathcal{Z}}(\mathscr{M})$. Evidently, it holds

$$
\mathscr{M}=\bigcap_{\mathscr{A} \in \mathrm{As} \mathscr{L}(\mathcal{M})} \mathscr{M}[\mathscr{P}] .
$$

where $\mathscr{M}[\mathscr{P}]$ denotes the $\{\mathscr{P}\}$-component of $\mathscr{M}$. It is easy to examine the family $\left\{\mathscr{M}[\mathscr{P}] ; \mathscr{P} \in \operatorname{Ass}_{\mathcal{L}}(\mathscr{M})\right\}$ satisfies all conditions in Corollary 6.4. The uniqueness is a result of the uniqueness of a reduced primary decomposition of $\mathscr{M}_{x}$ in $\mathscr{L}_{x}$ for each $x$ (see (2.1) and (2.3)). q.e.d.

Now we can prove the converse assertion of Corollary 3.5 for a special case.

Theorem 6.5. Suppose $X$ is a holomorphically complete complex space. Then a coherent sub-O-Module $\mathscr{M}$ of a coherent $\mathcal{O}$-Module $\mathscr{L}$ over $X$ is primary if aud only if the $H^{0}(X, \mathcal{O})$-submodule $H^{0}(X, \mathscr{M})$ of $H^{0}(X, \mathscr{L})$ is primary.

The "only if"-part is a result of Corollary 3.5. To see the "if"-part we need the following Lemma, which is a simple generalization of O. Forster [6], Lemma, p. 314.

Lemma 6.6. Under the same situation in Theorem 6.5, if $H^{\circ}(X, \mathscr{M})$ is primary, then an element $f \in H^{0}(X, \mathscr{L})$ with $f \in \mathscr{M}_{x}$ for some $x \in|\mathscr{L}| \subset \mathscr{K} \mid$ is contained in $H^{\circ}(X, \mathscr{M})$.

Proof of Theorem 6.5. Let $\mathscr{M}$ be a coherent primary $\mathcal{C}$-Module such that $H^{0}(X, \mathscr{M})$ is primary. Take a minimal element $\mathscr{P} \in \operatorname{Ass}_{\mathscr{L}}(\mathscr{M})$. The $\{\mathscr{P}\}$ component $\mathscr{M}[\mathscr{P}]$ is a coherent primary Module. To complete the proof, it suffices to see $\mathscr{M}=\mathscr{M}[\mathscr{P}]$. Even if $\mathscr{M} \neq \mathscr{M}[\mathscr{P}]$ we can easily find a point $x \in|\mathscr{L}| \mathscr{M} \mid$ with $\mathscr{M}_{x}=\mathscr{M}[\mathscr{P}]_{x}$. In view of Theorem 3.2 and Lemma 6.6, we can assert $H^{0}(X, \mathscr{M})=H^{0}(X, \mathscr{M}[\mathscr{P}])$. Now, Theorem 6.5 is an immediate consequence of Theorem $A$ for a holomorphically complete space. q.e.d.

Remark. In case that $X$ is holomorphically complete, Corollary 6.4 above is a special case of Theorem in [6] p. 328 .

7. Continuity of order of coherent Ideals over a complex space. As an application of the results in the previous sections, we can generalize Abhyankar's 
theorem concerning the continuity of order on a complex space. For this purpose, we recall some properties of ideals in a commutative ring.

Let $R$ be a commutative noetherian ring with identity. For a prime ideal $\mathfrak{p}$ in $R$ the symbolic $n$-th power $\mathfrak{p}^{(n)}$ of $\mathfrak{p}$ is defined by the equation $\mathfrak{p}^{(n)}:=$ $\mathfrak{p}^{n} R_{\mathfrak{p}} \cap R=\mathfrak{p}^{n}[\mathfrak{p}]$.

Firstly we give

Lemma 7.1. If an ideal $\mathfrak{A}$ in $R$ has a reduced primary decomposition

$$
\mathfrak{U}=\mathfrak{p}_{1} \cap \cdots \cap \mathfrak{p}_{s}
$$

and each $p_{i}$ is prime, then we have

$$
\mathfrak{U}^{n}[\mathbf{P}]=\mathfrak{p}_{1}^{(n)} \cap \cdots \cap \mathfrak{p}_{s}^{(n)} .
$$

for the isolated class $\mathbf{P}:=\operatorname{Ass}_{B}(\mathfrak{H})=\left\{\mathfrak{p}_{1}, \ldots, \mathfrak{p}_{s}\right\}$.

We shall denote $\mathfrak{A}^{n}[\mathbf{P}]$ by $\mathfrak{A}^{(n)}$ in the following.

Proof. As is easily seen, $\mathbf{P}$ is just equal to the class of all minimal elements of $\operatorname{Ass}_{R}\left(\mathfrak{A}^{n}\right)$. With both sides of the equation in question associate the same prime ideals $\mathfrak{p}_{1}, \ldots, p_{s}$. By $(2.3)$, it suffices to show for each $p_{i}$ the $\mathfrak{p}_{i}$-components of both sides coincide with each other. This follows from the equations

$\left(\mathfrak{A}^{n}[\mathrm{P}]\right)\left[\mathfrak{p}_{i}\right]=\mathfrak{U}^{n}\left[\mathfrak{p}_{i}\right]=\left(\mathfrak{A}^{n} R_{\mathfrak{p}_{i}}\right) \cap R=\left(\mathfrak{U} R_{\mathfrak{p}_{i}}\right)^{n} \cap R=\left(\mathfrak{p}_{i} R_{\mathfrak{p}_{i}}\right)^{n} \cap R=\mathfrak{p}_{i}^{(n)} \quad$ q.e.d.

Let $\mathfrak{A}$ be an ideal in $R$. By definition, for an arbitrarily given prime ideal $\mathfrak{p}$, the order $\operatorname{ord}_{\mathfrak{p}} \mathfrak{A}$ of $\mathfrak{U}$ at $\mathfrak{p}$ is the largest $n$ such that $\mathfrak{A} R_{\mathfrak{p}} \leqq \mathfrak{p}^{n} R_{\mathfrak{p}}$ in $R$, where $\operatorname{ord}_{\mathfrak{p}} \mathfrak{U}=0$ means $\mathfrak{A} \varsubsetneqq \mathfrak{p}$ and $\operatorname{ord}_{\mathfrak{p}} \mathfrak{U}=\infty$ means $a \cdot \mathfrak{U}=0$ for a suitable $a \notin \mathfrak{p}$.

Easily, we see

(7.2) If $\mathfrak{A} \supseteqq \mathfrak{Q}$, then $\operatorname{ord}_{\mathfrak{p}} \mathfrak{U} \leqq$ ord $d_{\mathfrak{p}} \mathfrak{L}$.

(7.3) If $\mathfrak{A}$ has a primary decomposition $\mathfrak{A}=\mathfrak{p}_{1} \cap \cdots \cap \mathfrak{p}_{s}$. with prime ideals $\mathfrak{p}_{i}$, then ord $\mathfrak{p}_{i} \mathfrak{X}^{(n)}=n$ for any $\mathfrak{p}_{i}$

Now, we come back to the study of coherent Ideals over a complex space $X$. For coherent Ideals we can apply all results in the previous sections with $\mathscr{S}=\mathbb{C}$

We consider the space $g(X):=\bigcup_{x \in|X|} \operatorname{Spec}\left(\mathscr{C}_{x}\right)$ or all irreducible germs of locally analytic subsets of $X$. According to H. Cartan [3], we can define the following canonical topology in $g(X)$. For an element $p \in g(X)$, say $\pi(p)=x$ 
for the canonical projection $\pi: g(X) \rightarrow X$, we choose a neighborhood $U$ of $x$ and a coherent prime Ideal $\mathscr{P}$ over $U$ with $\mathscr{P}_{x}=\psi$. And we consider the set $\mathfrak{u}(U, \dot{p})$ of all prime ideals associated with $\mathscr{P}_{y}$ for any $y \in U$. As a fundamental system of neighborhoods of $\mathfrak{p}$ we adopt the above sets $\mathfrak{l}(U, \mathfrak{p})$ with a parameter $U$. As is well known, $g(X)$ is a locally arcwise connected and locally compact Hausdorff space.

Take a coherent Ideal $\mathscr{A}$. For each $x \in|X|, \mathscr{A}$ defines an ideal $\mathscr{A}_{x}$ of $\mathscr{O}_{x}$. We can introduce the concept of the order ordp $\mathscr{A}$ of $\mathscr{A}$ at $p$ for each $\mathfrak{p} \in \mathfrak{g}(X)$ by the equality $\operatorname{ord}_{\mathfrak{p} \mathscr{A}}:=\operatorname{ord}_{\mathfrak{p}} \mathscr{A}_{x}$ in $\mathscr{C}_{x}$, where $x:=\pi(\mathfrak{p})$.

The following theorem is a generalization of Abhyankar's Theorem ([1] (11.3), p. 185).

THEOREM 7.4. For any coherent Ideal $\mathscr{A}$ ord $\mathscr{A}$ is continuous on $\mathfrak{g}(X)$.

For the proof, we need

LEMma 7.5. For a coherent Ideal $\mathscr{A}$ with $\operatorname{rad} \mathscr{A}=\mathscr{A}$, we can define a coherent Ideal $\mathscr{A}^{(n)}$ with the stalk $\left(\mathscr{A}^{(n)}\right)_{x}=\left(\mathscr{A}_{x}\right)^{(n)}$ at each $x \in|X|$.

Proof. This was proved by Kuhlmann ([9], Lemma 7, p. 401) for a special case. Using our results, we can easily prove this for a general case. Indeed, taking an isolated subset $\operatorname{Ass}_{\mathfrak{3}}(\mathscr{A})$ of $\operatorname{Ass}_{\mathfrak{3}}\left(\mathscr{A}^{n}\right)$, we put $\mathscr{A}^{(n)}=\mathscr{A}^{n}\left[\operatorname{Ass}_{\mathfrak{l}}(\mathscr{A})\right]$. By Theorem $6.3 \mathscr{A}^{(n)}$ is coherent and by Lemma $7.1 \mathscr{A}^{(n)}$ satisfies the desired condition.

$$
\text { q.e.d. }
$$

Proof of Theorem 7.4. The problem is local and each $\mathfrak{p} \in \mathfrak{g}(X)$ has a neighborhood $\tilde{\mathscr{P}}$ such that $\tilde{\mathscr{P}}=\bigcup_{y \in U} \operatorname{Ass}_{\partial_{y}}\left(\mathscr{P}_{y}\right)$ for a suitable neighborhood $U$ of $x:=\pi(\mathfrak{p})$ and a coherent prime Ideal $\mathscr{P}$ with $\mathscr{P}_{x}=\mathfrak{p}$. Therefore it suffices to show ordp $\mathscr{A}$ is constant on $\tilde{\mathscr{P}}$ above. Then, in view of Lemma 7.7, $\mathscr{P}^{(n)}$ is a coherent Ideal and it is primary over $U$ because of $\operatorname{Ass}_{\mathscr{O}}\left(\mathscr{P}^{(n)}\right)=\{\mathscr{P}\}$.

Now let $n_{0}:=\sup \left\{n ; \mathscr{A} \subseteq \mathscr{P}^{(n)}\right\}<\infty$. The proof of Theorem 7.4 is reduced to show ordp $\mathscr{A}$ is constantly equal to $n_{0}$ on $\tilde{\mathscr{P}}$. For each $\mathfrak{p} \in \tilde{\mathscr{P}}$ ord $\mathscr{A} \geqq n_{0}$ is evident by virtue of (7.2) and (7.3). On the other hand, applying Corollary 3.3 to a coherent primary Ideal $\mathscr{P}^{\left({ }^{(\eta)}\right.}$ and using Lemma 7.1, we can assert $\mathscr{A}_{y} \subseteq \mathfrak{p}^{\left(n_{0}\right)}$ and $\mathscr{A}_{y} \mathfrak{p}^{\left(n_{0}+1\right)}$ for any $\mathfrak{p} \in \tilde{\mathscr{P}}$ with $\pi(\mathfrak{p})=y \in U$. Assume $\operatorname{ord}_{\mathfrak{p}} \mathscr{A}_{y} \geqq n_{0}+1$ or $\mathscr{A}_{y} \cdot\left(\mathscr{O}_{y}\right)_{\mathfrak{p}} \subseteq p^{n_{0}+1}\left(\mathscr{O}_{y}\right)_{\mathfrak{p}}$ for some $\mathfrak{p} \in \mathscr{\mathscr { P }}_{\text {with }} \pi(\mathfrak{p})=y$. Then

$$
\mathscr{A}_{y} \subseteq \mathscr{A}_{y}\left(\mathscr{O}_{y}\right)_{\mathfrak{p}} \cap \mathscr{O}_{y} \leqq \mathfrak{p}^{n_{0}+1}\left(\mathscr{O}_{y}\right)_{\mathfrak{p}} \cap \mathscr{O}_{y}=\mathfrak{p}^{\left(n_{0}+1\right)} .
$$


This is a contradiction. We conclude $\operatorname{ord}_{p} \mathscr{A} \equiv n_{0}$ on $\tilde{\mathscr{J}}$.

Similarly, it is easy to show ordp $\mathscr{A} \equiv \infty$ on $\tilde{\mathscr{P}}$ if $\operatorname{ord}_{p} \mathscr{A}=\infty$ for some $\mathfrak{p} \in \tilde{g}$.

q.e.d.

8. Remarks on divisors on a complex space. Finally, we give some remarks on divisors on a reduced irreducible complex space.

By a non-negative divisor on a reduced irreducible complex space $X$ of dimension $n$, we mean a free sum $\sum \iota n_{\iota} V_{\iota}$ of $(n-1)$-dimensional irreducible analytic subsets of $X$ with non-negative integral coefficients such that the set $\left\{V_{\iota}: n_{\iota} \neq 0\right\}$ is a locally finite family of closed subsets of $|X|$. For an open subset $U$ of $X$ we denote the set of all non-negative divisors on $U$ by $\mathbf{D}^{+}(U)$. Taking another open set $U^{\prime}(\subseteq U)$, we can define the restriction mapping $r_{U U^{\prime}}$ : $\mathrm{D}^{+}(U) \rightarrow \mathrm{D}^{+}\left(U^{\prime}\right)$ by $r_{U U^{\prime}}\left(\sum_{\iota} n_{\iota} V_{\iota}\right)=\sum_{\iota} n_{\iota}\left(\sum_{\kappa} V_{\iota \kappa}\right)$, where $V_{\iota \kappa}$ runs through all irreducible components of $V_{\iota} \cap U^{\prime}$ and we put $n_{\iota}=0$ if $V_{\iota} \cap U^{\prime}=\phi$. If we call two elements $\alpha=\sum_{\iota} n_{\iota} V_{\iota}$ and $\beta=\sum_{\iota} m_{\iota} V_{\iota}$ in $\mathrm{D}^{+}(U)$ satisfying $n_{\iota} \leqq m_{\iota}$ for any ( to be $\alpha \leqq \beta, D^{+}(U)$ is considered as a partially ordered set. Obviously, the above $\gamma_{U U}$, is an order-reversing mapping.

According to Theorem 7.4, we can define the order of a coherent Ideal $\mathscr{A}$ over an open subset $U$ of $X$ for a coherent prime Ideal $\mathscr{P}$ over $U$ by $\operatorname{ord}_{\mathscr{A}} \mathscr{A}:=\operatorname{ord}_{\mathfrak{c}} \mathscr{A}_{x}$ for an arbitrarily fixed $\mathfrak{p} \in \bigcup_{y \in U} \operatorname{Ass}_{\mathscr{O}_{b}}\left(\mathscr{P}_{y}\right)$ with $\pi(\mathfrak{p})=x$. To each non-zero coherent Ideal $\mathscr{A}$ we assign an element $\varphi(\mathscr{A})=\sum_{V} \operatorname{ord} \mathscr{\mathscr { A } ( V )}$ $(\mathscr{A}) \cdot V$ in $\mathbf{D}^{+}(U)$, where $V$ runs through all irreducible analytic subsets of dimension $n-1$. On the other hand, there exists a canonical restriction mapping $r_{U C^{\circ}}$ of coherent Ideals over $U$ to $U^{\prime}$. Obviously, $\varphi$ is surjective. Indeed, an element $\sum_{\iota} n_{l} V_{\iota} \in \mathbf{D}^{+}(U)$ is the $\varphi$-image of a coherent Ideal $\cap_{\iota} \mathscr{F}\left(V_{l}\right)^{\left(n_{l}\right)}$.

In case of a normal complex space, we have the more precise information.

Theorem 8.1. If $X$ is a connected normal complex space of dimension $n$. The above mapping $\varphi$ gives a one-to-one order-reversing bijection between the class of all non-zero coherent Ideals $\mathscr{A}$ of $1 \cdot \operatorname{dim}_{\mathfrak{g}} \mathscr{A} \geqq n-1$ and $\mathrm{D}^{+}(X)$.

Proof. We have only to prove the injectivity of $\varphi$. As is well known, an ideal $\mathfrak{P}$ of $\operatorname{ldim} \mathfrak{N}=n-1$ in a normal ring is uniquely represented as the intersection of symbolic powers of the prime ideals associated with $\mathfrak{A}$. Therefore, if $X$ is normal, any coherent primary Ideal of height 1 is equal to a symbolic power $\mathscr{S}^{\prime}$ of a coherent prime Ideal $\mathscr{S}$ and any coherent Ideal of lower 
dimension $n-1$ is uniquely represented as the intersection of such primary Ideals. This shows two coherent Ideals $\mathscr{A}$ and $\mathscr{B}$ with $\varphi(\mathscr{A})=\varphi(\mathscr{B})$ coincide with each other.

q.e.d.

Corollary 8.2. Let $X$ be a connected normal complex space of pure-dimension $n$ and $X^{\prime}$ be its open subset. Suppose that any purely $(n-1)$-dimensional analytic subset of $X^{\prime}$ is uniquely continuable to the total space $X$. Then any coherent Ideal $\mathscr{A}$ with $1 . \operatorname{dim}_{\mathscr{B}} \mathscr{A}=n-1$ over $X^{\prime}$ is uniquely continuable to $X$.

Proof. By $\mathbf{A}(X)$ denoting the class of all coherent Ideals of lower dimension $n-1$, we have a commutative diagram

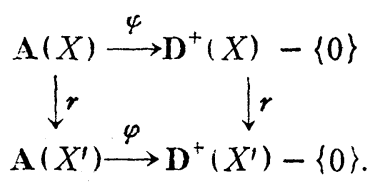

Since by the assumption $r_{X X},: \mathrm{D}^{+}(X) \rightarrow \mathrm{D}^{+}\left(X^{\prime}\right)$ is bijective and by Theorem 8.1 $\varphi^{\prime}$ s are also bijective, $r_{X X^{\prime}}: \mathbf{A}(X) \rightarrow \mathbf{A}\left(X^{\prime}\right)$ is bijective. This asserts Corollary 7.7 . q.e.d.

In case of a complex manifold, a coherent Ideal $\not$ of lower dimension $n-1$ is nothing but a holomorphic Cousin-II distribution. While, any Cousin-II distribution is represented as a quotient of two holomorphic Cousin-II distributions. The study of the continuability of Cousin-II distribution is reduced to that of coherent Ideals of lower dimension $n-1$.

Corollary 8.3. Under the same assumption in Corollary 8.2, if we assume, furthermore, $X$ is a complex manifold, then any Cousin-II distribution in $X^{\prime}$ is uniquely continuable to $X$.

Several kinds of conditions for the continuability of analytic subsets of codimension 1 were given in [5], [11] and [12] etc.. Immediately, we have the same conditions for the continuability of coherent Ideals and Cousin-II distributions.

\section{References}

[1] S. Abhyankar, Concepts of order and rank on a complex space and a condition for normality, Math. Ann., 141 (1960), pp. 171-192.

[2] N. Bourbaki, Fasc. XXVIIl. Algèbre commutativ. Chap. 4; Idéaux premiers associés et décomposition primaire, Paris, 1961. 
[ 3 ] H. Cartan, Seminaire, E. N. S., 1951/1952.

[ 4 ] H. Cartan, Seminaire, E. N. S., 130, 1960/1961.

[ 5 ] H. Fujimoto, On the continuation of analytic sets, J. of Math. Soc. Japan, 18 (1966), pp. 51-85.

[6] O. Forster, Primärzerlegung in Steinschen Algebren, Math, Ann., 154 (1964), pp. 307-329.

[7] H. Grauert, Ein Theorem der analytischen Garbentheorie und die Modulräume komplexer Strukturen. Publ. Math. I.H.E.S., No. 5, Paris 1960.

[8] A. Grothendieck, Eléments de Géometrie Algebrique, Publ. Math. I.H.E.S., No. 4, Paris 1960.

[ 9 ] N. Kuhlmann, Über die normalen Punkte eines komplexen Raumes, Math. Ann., 146 (1962), pp. 397-412.

[10] D. G. Northcott, Ideal theory, Cambridge Univ. Press, Cambridge, 1953.

[11] R. Remmert and K. Stein, Über die wesentlichen singularitäten analytischer Mengen, Math. Ann., 126 (1953), pp. 263-306.

[12] W. Rothstein, Zur Theorie der analytischen Mannigfaltigkeiten im Raum von $\mathbf{n}$ komplexen Veränderlichen, Malh. Ann., 129 (1955), pp. 96-138.

[13] G. Scheja, Fortsetzungssätze der komplexe analytischen Cohomologie und ihre algebraische Charakterisierung, Math. Ann., 157 (1964), pp. 75-94.

[14] W. Thimm, Lückengarben von kohärenten analytischen Modulgarben, Math. Ann., 148 (1962), pp. 372-394.

Nagoya Institute of Technology 